\title{
European Society for Child Communications of the European Society ESCAP for Child and Adolescent Psychiatry \\ and Adolescent Psychiatry
}

\section{Presidential column}

Dear colleagues,

The International Congress of ESCAP in Dublin (www. ESCAP2013.ie) is coming closer and closer (6-10 July 2013).

Many interesting abstracts are submitted. Many interesting speakers are invited.

The conference will be held at a newly built venue close to down town Dublin. If you want to enjoy the country before, during or after the conference you can visit the city and travel through wonderful Ireland.

During the past years, Child Psychiatry in and outside Europe was very productive in new research. We are happy that many top researchers and young talented researchers will present their work at this coming congress.

The last decennia an explosion of results were released in the international scientific literature (see European Child and Adolescent Psychiatry, this Journal), which are applicable in the treatment for children and adolescents with mental health problems. Much of these findings are translated into "protocols" that can be implemented in daily care for children and used by Physicians and Psychologists. These "protocols" create possibilities to design special "care programs" for children with different type of problems like ADHD, OCD, GTS, Depression, Anxiety Disorders, etc. based on care elements that are evidence based and very useful to help these children over a longer period of time.
The problem however is the implementation of these protocols on a large scale in the different European countries because of the differences in the organization of care between countries, within and between care centers, and many other (financial) problems. A large positive input might be given when over the boundaries of countries, care centers would cooperate to exchange their protocols and the experience with these protocols in daily use, in order to achieve more generally accepted evidence based "care programs".

The ESCAP Conference in Dublin 2013 will focus on these care programs under the heading of "Quality of Care". Clinicians from many different European countries will present how they implemented evidence based elements in their care programs and want to share and discuss their experience with the audience.

This will make the conference very interesting and useful for Clinicians, Psychiatrists, Psychologists and all others who play a role in the care for children.

The ESCAP conference will be the most important forum for exchanging skills and knowledge in child and adolescent psychiatry in Europe!

It will be a wonderful conference,

Ruud Minderaa, President of ESCAP

e-mail: r.b.minderaa@accare.nl 